\title{
Proposta de requisitos para um sistema de informação para a gestão do conhecimento em empresas nascentes de base tecnológica
}

Kalebe Luiz Pereira Benfica

kalebe.benfica@ufv.br

Adriana Ferreira de Faria

adrianaf@ufv.br

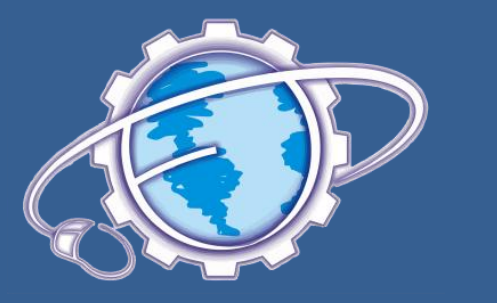

\section{RESUMO}

$O$ advento da microinformática e da internet proporcionou às empresas atuais profundos alicerces na tecnologia da informação por meio dos sistemas de informação, que permite armazenar, gerar e analisar enormes quantidades de dados, utilizando-os, posteriormente, para os processos de analise, tomada de decisão $e$ criação de novos conhecimentos. $O$ uso do conhecimento como agente promotor de inovação $e$ competitividade tem ganhado cada vez mais adeptos no mundo, assim a gestão do conhecimento assume um importante papel. Nesse contexto, destacam-se as empresas nascentes de base tecnológica de origem acadêmica (spin-offs acadêmicas), ricas em conhecimento e de caráter inovador, essas empresas transformam pesquisas acadêmicas em negócios. Para essas empresas, o Plano de Negócio, é fortemente atrelado ao planejamento da tecnologia e do produto, ou seja, o sucesso do negócio depende fortemente da gestão da inovação. Considerando essas características, a criação de sistemas de informação direcionados à gestão do conhecimento para este setor se revela de grande importância por auxiliar as empresas a manterem-se inovadoras. Dessa forma, o presente trabalho teve por objetivo geral identificar as principais características das empresas nascentes de base tecnológica a fim de propor os requisitos de um sistema de informação que atenda as principais necessidades dessas empresas para a gestão do conhecimento e da inovação.

Palavras-chave: Sistemas de informação; Gestão do conhecimento; Empresas nascentes de base tecnológica.

\section{Proposal for information system requisites for knowledge management in new technological based business enterprises}

\begin{abstract}
The arrival of microcomputer science and the internet provided to the existing business enterprises sound information technology through information systems, which allow to store, generate, and analyze great data quantities, using them later for analysis processes, decision taking, and creation of new knowledge. The use of knowledge as an innovation promoting agent has gained many supporters in the world. This way, knowledge management assumes an important role. In this context, the sturt up enterprises of academic origin stand out (academic spin-offs, rich in knowledge and having an innovative character, these business enterprises transform academic research into business. For these organizations, the business plan is strongly linked to technology and the product planning, that is, the business success depends strongly on the innovation management. In regard to these characteristics, the creation of information systems directed towards knowledge management reveals itself as being of great importance to help the business enterprises remain innovative. Thus, this study has the objective to identify the main characteristics of the sturt up, in order to propose the requisites of an information system which addresses the main needs of these businesses for innovation and knowledge management.
\end{abstract}

Key words: Information system; Knowledge management; New technological based business enterprises. 


\section{Introdução}

$\mathrm{O}$ advento da microinformática e da internet juntamente com a evolução das tecnologias de informação possibilitou o armazenamento e a gestão de grandes quantidades de dados e sua difusão por meio das organizações, culminando no surgimento de um novo paradigma gerencial baseado na utilidade estratégica de informações. $\mathrm{O}$ conhecimento derivado da gestão destas informações pode ser considerado uma forma de capital presente nas organizações (ZABOT \& SILVA, 2002).

$\mathrm{O}$ favorecimento ao surgimento de inovações e a maior flexibilidade perante as mudanças do ambiente são benefícios alcançados com o uso estratégico de informações, sendo considerados fatores decisivos para a sobrevivência das empresas no contexto atual. Empresas norteamericanas, entre os anos de 1965 a 1991, passaram de um gasto de 18,2 bilhões anuais para 112 bilhões anuais em tecnologias de informações, ultrapassando os gastos com maquinário de produção (ALVARENGA NETO, 2008).

Neste contexto, destacam-se as empresas nascentes de base tecnológicas (ENBT's), também conhecidas como spin-offs acadêmicas. Estas empresas se caracterizam pela exploração da propriedade intelectual desenvolvida nas universidades, transformando o conhecimento em riquezas (REIS, 2005). Para as spin-offs acadêmicas, o Plano de Negócio é fortemente dependente do planejamento da tecnologia e dos produtos. Dessa forma, para essas empresas, a gestão do conhecimento para á inovação torna-se ferramenta estratégica capaz de auxiliar na promoção do sucesso.

De acordo com Laudon \& Laudon (2004), o conhecimento se destaca como um patrimônio essencial e estratégico, sendo que o sucesso organizacional depende cada vez mais da capacidade da empresa de gerenciar este conhecimento (). Se esse fato é verdade para as grandes empresas, ele torna-se crucial para as spinoffs acadêmicas, que em sua grande maioria surgem como pequenas empresas, em um mercado dominado por players considerados "agressivos" e muitas vezes, "predadores".

Considerando estas premissas, destaca-se a necessidade de criação de sistemas de informação direcionados à Gestão do Conhecimento em
Empresas Nascentes de Base Tecnológicas devido à escassez de ferramentas e publicações especificas para esse setor. Neste contexto, este trabalho teve como objetivo geral identificar as principais características das empresas nascentes de base tecnológica a fim de propor os requisitos de um sistema de informação que atenda as principais necessidades dessas empresas para a gestão do conhecimento e da inovação. O trabalho considerou pesquisas e estudos realizados sobre o ambiente em que estas empresas se encontram, as suas principais características e os tipos de ferramentas disponíveis no mercado para a gestão do conhecimento.

\section{Revisão de literatura}

\subsection{Gestão do conhecimento e tecnologias da informação}

A gestão do conhecimento (GC) pode ser definida como sendo uma forma de gerenciar o capital intelectual presente dentro das organizações (STEWART, 1998). Segundo Amaral (2008), a gestão do conhecimento também pode ser vista como um programa ou um conjunto de ações que visam aumentar continuamente a competência dos funcionários e a eficiência dos processos de negócio, por meio de ações que fomentem um ambiente de estimulo a aprendizagem e a criação de comunidade de prática.

Ambos os autores definem a GC como sendo um processo gerencial focado em uma cultura organizacional voltada ao estimulo a aprendizagem e a gestão do capital intelectual. Porem a definição destes autores não engloba aspectos envolvidos com a sistematização destes processos envolvendo a Tecnologias de Informação (TI) como apoiadora destes processos.

Neste contexto, o conceito de a gestão do conhecimento como sendo um conjunto de atividades relacionadas a geração, codificação e transferência do conhecimento é mais adequada (DAVENPORT \& PRUSAK 1998). Uma segunda definição classifica a gestão do conhecimento como sendo um processo sistêmico de coleta, organização, análise e compartilhamento do conhecimento (FALCÃO \& BRESCIANI FILHO, 1999).

De acordo com as definições acima, as tecnologias de informação influenciam o processo de gestão do conhecimento, ampliando o alcance e 
acelerando os fluxos de informações. As ferramentas de gestão do conhecimento auxiliam o processo de captura, estruturação e disponibilização do conhecimento. $\mathrm{O}$ objetivo principal das ferramentas de Gestão do Conhecimento é modelar parte do conhecimento que existe na cabeça das pessoas e nos documentos corporativos, disponibilizando-os para toda a organização (DAVENPORT \& PRUSAK, 1998).

É importante ressaltar que independente do uso destas ferramentas, um processo de GC não pode descartar os aspectos humanos e culturais presentes na organização. Neste contexto, a gestão do conhecimento se mostra uma combinação complexa que envolve pessoas, processos e tecnologia, logo para se implantar um sistema de Gestão do Conhecimento, o suporte tecnológico se faz necessário, mas não é a única variável (CARVALHO, 2006).

No âmbito da gestão do conhecimento, a tecnologia atua na construção de novos conhecimentos influenciando qualitativamente $\mathrm{e}$ quantitativamente esse processo e ao mesmo tempo abre portas para novas características e possibilita a formação de comunidades de conhecimento (CASTRO, 1999). Logo, a tecnologia se transforma em uma poderosa ferramenta para suporte a Gestão do Conhecimento dentro das empresas. Ainda para os autores, são ferramentas de Gestão do Conhecimento aquelas que dão suporte pelo menos a um dos conjuntos de atividades para a geração, codificação ou transferência de conhecimento.

Segundo Carvalho (2000), é possível identificar três estágios da infraestrutura de tecnologia da informação com orientação para o conhecimento, a saber:

- $1^{\circ}$ Estágio - Sistemas de Informação Tradicionais: os sistemas capturam, armazenam e recuperam informações dentro de um determinado contexto.

- $2^{\circ}$ Estágio - Contextualização: são catalogados dados a respeito de onde uma informação está armazenada e quem a acessa, que processos fazem uso da mesma e quais clientes ou produtos a informação está relacionada. O conteúdo ganha atributos de contexto na medida em que vai sendo utilizado.

- $3^{\circ}$ Estágio - o contexto direciona a distribuição do conteúdo. O sistema proativamente estabelece elos entre as informações relevantes.

Existem alguns aspectos comuns entre os diferentes tipos de ferramentas utilizados na Gestão do Conhecimento. Estes aspectos, ou padrões, servem como base para a classificação destas ferramentas de acordo com as suas funcionalidades principais. O objetivo básico de uma ferramenta de Gestão do Conhecimento é permitir a busca, a organização e a disseminação do conhecimento. Ela deve fornecer um ambiente que permita aos usuários acessar, administrar e manter o conteúdo de um modo organizado. O conteúdo é o capital intelectual proprietário de maior validade para uma organização (CARVALHO, 2000).

As ferramentas voltadas a GC podem ser agrupadas nas seguintes categorias: sistemas especialistas e de inteligência artificial, repositórios de conhecimento amplo, sistemas do conhecimento em tempo real, ambientes de conhecimento voltados para a Web e sistemas de análise de prazo mais longo (DAVENPORT \& PRUSAK, 1998).

A classificação apresentada concentra-se nas ferramentas voltadas ao trabalho colaborativo e à preservação da memória organizacional, mas não incluem as ferramentas de apoio à inovação que são extremamente úteis no processo de geração de conhecimento. Sendo assim, uma categorização alternativa classifica as ferramentas de GC em oito categorias, relacionadas por características comuns, como a sua funcionalidade essencial, o processo de Gestão do Conhecimento (geração, codificação e transferência) adotado, o tipo de conhecimento (tácito ou explicito) e por fim a área de origem dos conceitos. Estas categorias são (CARVALHO, 2000):

- Ferramentas voltadas para a intranet: Baseadas em redes computacionais, a intranet é um termo utilizado para descrever uma rede coorporativa privada de computadores que se baseia em padrões de comunicação semelhantes aos utilizados pela internet e tem como objetivo principal promover a comunicação dentro das empresas. A intranet é o ambiente de trabalho ideal para o compartilhamento de informações dinâmicas e interligadas. São de grande valia na codificação e transferência do conhecimento tácito e explicito.

- Sistemas de GED (Gerenciamento Eletrônico de Documentos): Os sistemas de GED são repositórios de importantes documentos corporativos e atuam como armazéns do conhecimento explícito e se caracterizam como sistemas repositórios de conhecimento explícito estruturado.

- Sistemas de groupware: Groupware consiste no software projetado para auxiliar grupos de pessoas, geralmente distantes fisicamente, mas 
que trabalham em conjunto. O groupware se propõe a aumentar a cooperação e a comunicação interpessoal com base em fortes apelos sociais e organizacionais. Permite que grupos de pessoas exerçam atividades conjuntamente em busca de um único objetivo e possibilita a ocorrência de todos os processos de gestão do conhecimento.

- Sistemas de workflow: Com sua origem em organização de sistemas e métodos, é um sistema informatizado que oferece suporte para processos padronizados de negócio. $\mathrm{O}$ workflow é uma ferramenta que auxilia na explicitação do conhecimento tácito que se encontra embutido nos processos, codifica as regras de negócio e identifica como as pessoas trabalham em conjunto para dar andamento a um processo organizacional.

- Sistemas para construção de bases inteligentes de conhecimento: $\mathrm{O}$ conceito de inteligência encontra-se associado aos conceitos de habilidade, aptidão, adaptação, aprendizagem e raciocínio. Sistemas para construção de bases inteligentes de conhecimento são sofisticados, abrangem todos os processos da Gestão do Conhecimento e permitem o compartilhamento de suas duas dimensões.

- Business intelligence: Sistemas que permitem as empresas encontrar, em meio à sua base de dados, as informações fundamentais sobre $\mathrm{o}$ seu negócio, podendo assim antecipar tendências, se adiantar no lançamento de produtos, conhecer melhor os seus clientes e alavancar seu potencial competitivo. Permitem a análise de dados e buscam extrair novos conhecimentos com base nestas analises, logo codifica e compartilha o conhecimento explicito presente nos bancos de dados das empresas.

- Sistemas de mapa de conhecimento: Sistemas que foram projetados desde a sua primeira versão com o foco na Gestão do Conhecimento. Uma característica das ferramentas específicas é o foco no uso da informação com pouca estrutura para apoiar o processo de criação do conhecimento. Essas ferramentas oferecem apoio ao trabalho cooperativo e ao compartilhamento de conhecimento explicito e tácito.

- Ferramentas de apoio à inovação: Essa categoria enquadra ferramentas de Gestão do
Conhecimento que apoiam a geração de novos conhecimentos, contribuindo assim para a inovação tecnológica. Boa parte das iniciativas de Gestão do Conhecimento envolve o compartilhamento de melhores práticas, ou seja, de algo que já é conhecido. Devido a sua natureza desenvolvedora, abrangem todos os processos da Gestão do Conhecimento e permitem o compartilhamento de suas duas dimensões.

\subsection{Empresas Nascentes de Base Tecnológica}

Empresas Nascentes de Base Tecnológica (ENBT) podem ser consideradas como resultado do empreendimento da geração de ideias e produtos de base tecnológica, o chamado empreendedorismo tecnológico. Por sua vez, este é caracterizado pela geração de empresas nas quais os sócios fundadores possuem formação acadêmica e/ou experiência profissional em pesquisa e desenvolvimento (P\&D), geralmente nas áreas de ciências aplicadas ou engenharia.

O empreendedorismo tecnológico surge como uma das principais formas de levar os resultados de pesquisa para o mercado. Esse tipo de empreendedorismo em particular envolve a geração de novas empresas por empreendedores com experiências anteriores em atividades de $P \& D$, originados de grandes empresas ou de ambientes acadêmicos (DRUMMOND, 2005).

Os empreendimentos gerados a partir deste processo de transferência tecnológica são denominados spin-off's empresariais. Quando a organização fonte da tecnologia é uma empresa privada, os novos negócios são chamados de spinoff's corporativos. Entretanto, esta tecnologia pode ser proveniente também das universidades a partir dos resultados de pesquisa alcançada anualmente nos laboratórios acadêmicos. Nesses casos as novas ENBT's são denominadas spin-off's acadêmicas (DRUMMOND, 2005). O principal objetivo destas organizações é a exploração comercial do conhecimento ou da propriedade intelectual desenvolvidos nas universidades.

$O$ processo de geração das spin-offs acadêmicas pode ser dividido em quatro etapas: conscientização, pré-incubação, incubação e implementação da empresa. A primeira etapa visa conscientizar alunos e professores para a importância de gerar valor econômico a partir das pesquisas acadêmicas. A segunda abrange o estudo 
da viabilidade técnica e comercial do projeto de base tecnológica. A terceira compreende o amadurecimento do projeto, nos dois primeiros anos de vida, normalmente dentro de uma incubadora de empresas. E a quarta, por sua vez, corresponde à consolidação da empresa já inserida de forma independente no mercado (CHENG et al., 2004).

Durante este processo de geração se destaca a fase de pré-incubação como sendo determinante para a sobrevivência futura da organização. É durante esta fase que tópicos importantes para o planejamento da empresa são abordados. Documentos como o plano de negócios e o plano tecnológico definirão como a incorporação das tecnologias será feita aos protótipos e produtos, além de definirem também o programa de lançamento de produtos e como estes sustentarão a empresa financeiramente (CHENG et al., 2004). Este planejamento tecnológico, muitas vezes realizado na pré-incubação, fornece ao empreendedor subsídios importantes para a reflexão sobre aspectos vitais e importantes do empreendimento e provê o empreendedor de informações baseadas em estudos de viabilidade pertinentes à criação do negócio, que o auxiliarão desde a identificação de uma oportunidade (ideia) até a finalização do projeto ou lançamento do produto (LEONEL, 2007).

Neste contexto, o planejamento inicial visa proporcionar uma compreensão dos aspectos comerciais, financeiros e organizacionais juntos aos demais aspectos do desenvolvimento tecnológico. Este planejamento se dá pela elaboração de um plano de negócios e de um plano tecnológico. Plano tecnológico é um documento formalizado que contem informações importantes na estruturação do novo negócio e as diretrizes do processo de desenvolvimento de produtos pela empresa. Como resultado, espera-se que a empresa consiga desenvolver protótipos que demonstrem o funcionamento da tecnologia e que esta possa ser estendida a produção industrial (LEONEL, 2007).

As informações contidas no plano tecnológico estão integradas de modo que permita uma visão melhor dos aspectos envolvidos no processo de implantação de uma nova empresa. Estes aspectos são basicamente a tecnologia desenvolvida, o produto que será comercializado e o mercado no qual a empresa irá se posicionar. Juntos, esses três aspectos compõem o que na literatura é chamado de trinômio tecnologia, produto mercado (TPM). A compreensão deste trinômio é de vital importância para o sucesso e permanência das ENBT's no mercado. A compreensão da inter-relação do TPM permite a empresa direcionar o seu processo de desenvolvimento de tecnologias e produtos para o mercado e seus potenciais clientes de forma que os produtos comercializados atendam efetivamente uma necessidade deste mercado e gere retorno financeiro para a empresa (DRUMOND, 2005).

O plano tecnológico contempla informações relativas a diversas áreas do conhecimento. Estas informações são geradas em equipes de trabalho multidisciplinares, envolvendo profissionais exclusivamente técnicos e voltados à pesquisa e desenvolvimento com profissionais com competências gerenciais que permitem a visualização do mercado e suas necessidades (REIS, 2005). Neste contexto, a figura do empreendedor surge como sendo de suma importância na garantia de integração da equipe de trabalho durante a evolução das atividades de desenvolvimento e o sucesso das ENBT's em seus primeiros anos de vida está bastante relacionado aos focos tecnológico e mercadológico dado pelos empreendedores ao desenvolvimento de seus produtos e que o envolvimento dos empreendedores com essas questões, desde os primeiros passos da ENBT, é apontado como fundamental para levar as tecnologias com sucesso dos laboratórios até o mercado (DRUMMOND, 2005).

Em ambientes acadêmicos, a importância dessa integração torna-se ainda mais visível tendose em vista a inexperiência dos empreendedores com relação às questões de mercado, pois em sua grande maioria, estes empreendedores são pesquisadores atuantes em áreas de conhecimento técnico. É fato que os pesquisadores empreendedores, devido a sua origem acadêmica, tendem a ter apenas uma visão da tecnologia, negligenciando algumas vezes os outros pontos de vista. Logo, a formalização de uma ferramenta de gestão que reúna informações estratégicas para o futuro gerenciamento da empresa e que auxilie na orientação do negócio sem deixar de lado as perspectivas referentes à tecnologia, produto e mercado são de vital importância no gerenciamento da empresa.

Estas informações devem abranger o planejamento organizacional, de mercado, produção e financeiro sem deixar de levar em consideração as tecnologias que serão embarcadas nos produtos e suas informações técnicas. No contexto organizacional, deve-se levar em conta informações 
que ajudem no planejamento e desenvolvimento da equipe de trabalho, como competências individuais e experiências profissionais. Quanto ao mercado, informações advindas de análises de mercado e estratégias de marketing devem expressar o conhecimento do mercado consumidor relativos à segmentação, crescimento, características dos consumidores, concorrência, participação de mercado dos concorrentes, estratégias de vendas que envolvam técnicas de comercialização, dos aspectos regulatórios existentes que podem incluir legislações, certificação e registros e por fim informações acerca do diferencial do produto, políticas de preço, clientes, distribuição e estratégias de publicidades. Em relação à produção, devem-se conter informações sobre o sistema produtivo, dos processos produtivos, índices de refugos, parâmetros de controle do processo (DRUMMOND, 2005).

Por fim, informações financeiras devem abranger a relação de todas as decisões tomadas pelos empreendedores com seus respectivos impactos financeiros na empresa bem como os recursos econômicos disponíveis. É importante destacar que análises financeiras dependem fortemente da qualidade das informações obtidas sobre os outros fatores, como o de mercado e as projeções realizadas. Como exemplo de análises financeiras utilizadas é possível citar os demonstrativos de fluxo de caixa, análises de ponto de equilíbrio e demonstrativos de resultados (DRUMMOND, 2005).

Em relação às informações tecnológicas e de produto consideram-se a caracterização da tecnologia e dos produtos, o estágio de desenvolvimento, capacidade técnica da equipe, ciclo de vida, barreiras e riscos, oportunidades inicialmente vislumbradas e outras oportunidades (corresponde a gestão de plataforma) formalizado no plano tecnológico. Estes tópicos abordados correspondem aos estudos de viabilidade técnica e econômico-financeira, fazendo assim o papel de "trilho" entre a tecnologia e sua comercialização no mercado. $\mathrm{O}$ pesquisador atual necessita interagir mais com o mercado para entender a dinâmica da inovação existente dentro do setor industrial onde está localizado seu negócio. Nessa dinâmica é essencial compreender a relação entre as tecnologias do produto e dos processos de manufatura, além da organização da corporação e a estrutura da indústria, para garantir que $\mathrm{o}$ empreendimento gerado pela spin-off acadêmica seja o primeiro a explorar a nova tecnologia ou produto (REIS, 2005).

\section{Metodologia}

A proposta deste trabalho é identificar as características das empresas nascentes de base tecnológica a fim de propor os requisitos de um sistema de informação que atenda as principais necessidades dessas empresas para a gestão do conhecimento e da inovação.

O sistema deve armazenar e disponibilizar as informações de um modo que possam ser utilizadas no dia-dia da empresa como um apoio na formulação de estratégias no desenvolvimento de produtos, tecnologias e conhecimentos. Com esta ferramenta, será possível a integração das diferentes funções envolvidas no processo de desenvolvimento de produto, fazendo uma ligação entre as áreas técnicas que trabalham efetivamente no desenvolvimento da tecnologia com as informações necessárias para o direcionamento estratégico desse desenvolvimento, orientado para o mercado.

Para o desenvolvimento desse trabalho, foram desenvolvidas as seguintes atividades:

- Revisão bibliográfica sobre os temas: Gestão do Conhecimento, Gestão de Projetos, Sistemas de Informação, Tecnologias de Informação.

- Identificação de tecnologias de informação disponíveis no mercado para o trabalho de gestão do conhecimento nas empresas nascentes de base tecnológica.

- Proposição dos requisitos da ferramenta.

\section{Resultados: requisitos do sistema para a gestão do conhecimento}

Com base nos três estágios de infraestrutura tecnológica, identificados na revisão de literatura deste trabalho, os requisitos sugeridos buscam atender uma série de aspectos funcionais e técnicos presentes em sistemas de informação com foco na gestão do conhecimento. Esses aspectos podem ser resumidos rapidamente em mecanismos de busca e pesquisa disponíveis, distribuição de conteúdo aos usuários de acordo com critérios pré-definidos, categorização e sistematização de informações, acessibilidade via web e por fim sua flexibilidade mediante aos diferentes casos de uso, conforme cita 
CARVALHO (2000). A seguir estão listados os requisitos propostos para uma ferramenta de gestão do conhecimento em ENBT's.

Registrar e classificar informações integradamente: $o$ sistema deve oferecer informação estruturada que possa servir de apoio à tomada de decisão. Estas informações devem estar disponíveis e relacionadas por áreas de interesse e classificadas de acordo sua natureza, sendo estas informações referentes ao desenvolvimento da tecnologia, do produto e dos mercados abordados. Este tipo de informação pode ser gerada em reuniões de equipe, contato com os consumidores e parceiros, lições aprendidas (relatórios e depoimentos), ideias, pesquisas em bancos de dados públicos e privados referentes a publicações importantes, URL's, documentos e projetos de P\&D. Essas informações devem estar relacionadas com os tópicos importantes para o processo de desenvolvimento de tecnologias e produtos.

Priorizar informações: $o$ sistema deve priorizar as informações com em sua importância e qualidade. Informações possuem diferentes graus de importância estratégica para empresa e diferentes níveis de confiabilidade, logo é importante identificar aquelas que mais contribuem para o cumprimento da estratégia empresarial e que possuam maior grau de confiança ou exatidão.

Inserir gaps: $\mathrm{O}$ sistema deve possibilitar aos usuários explicitar as lacunas de conhecimento, informações e recursos que interferem no processo de desenvolvimento de produtos da empresa.

Permitir identificar o estágio de desenvolvimento de cada projeto: Um projeto, nesse caso, corresponde basicamente no desenvolvimento de uma ou mais tecnologia que possa ser embarcada em um produto com a finalidade de atender uma demanda de mercado condizente com a estratégia empresarial, ou desenvolvimento do produto. Ao usuário deve ser permitida a visualização do estágio de desenvolvimento da tecnologia ou do produto, que podem estar em estágio de pré-desenvolvimento, desenvolvimento, ou pós-desenvolvimento. Em todos estes estágios existem informações estratégicas para o sucesso de mercado do produto que devem ser gerenciadas.

Ser flexível: O sistema dever ser flexível e genérico, ou seja, apresentar uma estrutura que se adapte aos mais variados setores produtivos.

Capturar e organizar ideias: o sistema deve ser concebido considerando ferramentas como mapas mentais e fóruns, para externalizar informações tácitas dispersas no ambiente. A utilização do fórum permite aos integrantes da empresa expressar suas opiniões sobre determinado assunto, podendo através da utilização de mapas mentais serem organizadas de forma que gerem algum retorno para empresa. $O$ tema e os integrantes do fórum também podem ser previamente definidos ou abertos para todos, desta forma pode-se utilizá-lo como uma "sala de reunião" online.

Criar mapas estratégicos: o sistema deve criar mapas estratégicos, adequando e organizando as informações relativas a recursos disponíveis, tecnologia, produto e mercado, bem como o horizonte temporal (período de desenvolvimento). O sistema deve ser capaz de visualizar graficamente por meio de um roadmap a evolução do desenvolvimento de produtos e tecnologias.

Criar planos de ação: O sistema deve fornecer subsídios para criar ou armazenar planos de ação quando necessários. Os mesmo devem estar associados a um ou mais responsáveis e suas áreas de atuação.

Possibilitar acesso via web: o usuário do sistema deve ser capaz de acessar o sistema em qualquer lugar, bastando apenas uma conexão de internet e um navegador web.

Possibilitar a comunicação assíncrona e síncrona: os usuários devem ser capazes de se comunicarem por meio de mensagens instantâneas (síncrono), e-mails ou notas relacionadas com o interesse (assíncrono).

Rastrear e identificar usuários: o sistema dever permitir a construção de um banco de dados que contenha o perfil atualizado de cada usuários do sistema, setor de serviços, especialidade e suas atribuições e responsabilidades no processo de desenvolvimento de produto ou seja, uma espécie de páginas amarelas contendo informações sobre especialistas e usuários do sistema. Mudanças, atualizações ou incremento de informações no sistema devem estar relacionados a quem as executou, hora e data.

Apresentar controle de usuários: o sistema de vê permitir o acesso às informações e a utilização do sistema de acordo com o nível de permissão de cada usuário, definido por um administrador do sistema. Ao administrador serão atribuídas todas as funcionalidades do sistema, tendo ele o poder de realizar qualquer mudança e alteração. Aos usuários será possível a customização da conta, de acordo com a necessidade de cada função. 
Banco de dados com área de trabalho compartilhada: a área compartilhada é destinada ao armazenamento do conhecimento explícito da empresa. Estes conhecimentos podem se apresentar na forma de arquivos de texto, planilhas, gráficos e outros. O trabalho compartilhado ajuda no controle de versões e na visualização da evolução de ideias e discussões. Quando um arquivo for alterado, automaticamente os interessados são avisados e comentários podem ser realizados.

Busca: o sistema de permitir a realização de buscas de conteúdo, de acordo por palavras chaves com os tipos de informações contidas no sistema: documentos, relatórios, e-mails, notas, notícias, perfis e geral (busca em todo o conteúdo).

Gestor de dados e informação: o sistema deve possuir mecanismos que façam a classificação, o arquivamento e a busca dos dados e das informações contidas no sistema. Esses mecanismos devem ser capazes de disponibilizar a informação em forma visual e textual, dependendo da necessidade e do tipo de informação.

Templates: o sistema deve permitir a introdução de templates de relatórios, padrões de documentos, planilhas e planos de ação de acordo com a necessidade de cada empresa.

Mapas mentais: o sistema deve possibilitar a geração e o registro de ideias, lições aprendidas, resultado de reuniões, não deixando disperso nenhum tipo de informação relevante.

Fóruns, listas de discussões e ferramentas de apoio a reuniões: por meio dessas ferramentas, o sistema deve auxiliar no fluxo e disseminação de informações, ideias e lições aprendidas e oferece apoio para planejar e agendar reuniões. Em listas de discussões é possível colocar determinados assuntos em discussão e assim votar pela melhor opção, que após encerrada ficará armazenada no sistema.

Clipping: o sistema deve possuir uma área destinada ao compartilhamento de notícias publicadas em jornais, revistas e sites que estejam de alguma forma relacionada com interesses da empresa.

\section{Considerações Finais}

O conhecimento bem gerenciado é um fator competitivo que pode influenciar na permanência ou não da empresa no mercado. A busca por um sistema de informação que atenda esta necessidade em ENBT's se torna de grande relevância dado a sua característica de ser inovadora. O fato é que apesar do grande potencial tecnológico e de desenvolvimento existente nestas organizações, estas, em sua grande maioria, são pequenas empresas, para as quais existem poucas soluções tecnológicas oferecidas no mercado a um custo acessível.

Grandes empresas desenvolvedoras de softwares concentram seus esforços em desenvolvimento de sistemas de informações muitas vezes complexos, caros e difíceis de serem implantados. Ferramentas assim acabam ficando longe da realidade das pequenas empresas e por consequência disto, longe das empresas nascentes de base tecnológica.

Dessa forma, considerando o exposto acima e a importância da gestão do conhecimento e da inovação para as spin-offs acadêmicas, esse trabalho se propôs a identificar os requisitos de um sistema de informação para essa finalidade. Com os requisitos aqui identificados, como trabalho futuro espera-se desenvolver o sistema proposto.

\section{Agradecimentos}

Deixo expresso os meus profundos agradecimentos a Fundação de Amparo à Pesquisa do estado de Minas Gerais - FAPEMIG pelo apoio e investimento durante o ano de 2011 na realização desta pesquisa e que possibilitou a elaboração deste artigo.

\section{Referências}

ALVARENGA NETO, R. C. D. D. Gestão do Conhecimento em Organizações. São Paulo: Saraiva, 2008

AMARAL, D. C. O papel da gestão do conhecimento no processo de desenvolvimento de produtos. 2008

CARVAlHO, R. B. Aplicações de Software de Gestão do Conhecimento: Tipologias e Usos. Dissertação (Programa de Pós-Graduação em Ciência da Informação) - Universidade Federal de Minas Gerais. Belo Horizonte. 2000.

CASTRO, M. N. M. Aprendizagem na organização e novas tecnologias aplicadas à educação à distância. Belo Horizonte: Programa de Pós-Graduação em Ciência da Informação, 1999.

CHENG, L. C.; DRUMMOND, P.; MATTOS, P. A integração do trinômio tecnologia, produto $\mathrm{e}$ 
mercado na pré- incubação de uma empresa de base tecnológica, 2004

DAVENPORT, T. H.; PRUSAK, L. Conhecimento empresarial. Rio de Janeiro: Campus, 1998.

DRUMMOND, P. H. F. O planejamento tecnológico de uma empresa de base tecnológica de origem acadêmica por intermédio dos métodos technology roadmapping (TRM), technology stage-gate (TSG) e processo de desenvolvimento de produtos (PDP) tradicional. Dissertação (Programa de Pós-Graduação em Engenharia de Produção) - Universidade Federal de Minas Gerais, Belo Horizonte. 2005.

FALCÃO, S. D.; BRESCIANI FILHO, E. Gestão do conhecimento. Revista da III $^{\mathrm{a}}$ Jornada de Produção Científica das Universidades Católicas do Centro-Oeste, Goiânia, 1999.

LAUDON, Kenneth C.; LAUDON, Jane P. Sistemas de Informação Gerenciais. Administrando a empresa digital. São Paulo: Prentice Hall, 2004.

LEONEL, S. G. Um estudo do processo de planejamento tecnológico de uma empresa nascente: alinhando tecnologia, produto e mercado com foco na necessidade do cliente. 2007. Dissertação (Programa de Pós- Graduação em Engenharia de Produção) - Universidade Federal de Minas Gerais, Belo Horizonte. 2007.

REIS, L. P. Revelando as fases do planejamento tecnológico e a atribuição de funções entre os membros da equipe empreendedora no contexto da gestão de desenvolvimento de produtos (gdp) em empresas iniciantes de base tecnológica (ebt's). Monografia (Graduação em Engenharia de Produção) - Universidade Federal de Minas Gerais, Belo Horizonte. 2005.

STEWART, T. Capital Intelectual - A nova vantagem competitiva das empresas. Rio de Janeiro: Campus, 1998.

ZABOT, J. M.; SILVA, L. C. Gestão do conhecimento: aprendizagem e tecnologia, construindo a inteligência coletiva. São Paulo: Atlas, 2002.

\section{Artigo selecionado entre os 10 melhores do VIII Encontro Mineiro de Engenharia de Produção - EMEPRO 2012.}

